\title{
RISE/FALL TIME ENHANCEMENT OF THE SPALLATION NEUTRON SOURCE LINAC LEBT CHOPPER SYSTEM *
}

\author{
Vladimir V. Peplov, Robert B. Saethre, \\ Oak Ridge National Laboratory (ORNL), \\ Spallation Neutron Source (SNS) \\ 1 Bethel Valley Road, Oak Ridge, TN 37831 USA
}

\begin{abstract}
The Spallation Neutron Source (SNS) Linac Low Energy Beam Transport (LEBT) chopper system provides fast chopping of the $\mathrm{H}^{-}$ion beam in the LEBT structure. Four identical pulsed power supplies (pulsers) create a series of $\pm 2.5 \mathrm{kV}$ pulses to the four deflection electrodes floating on the focusing voltage of $-50 \mathrm{kV}$. Each pulser is connected to the electrode through the network which consists of high voltage (HV) cables, a blocking capacitor, HV feed-through connectors, current-limiting resistors and transient voltage suppressors. Effective beam chopping requires minimal rise/fall time of the rectangular HV pulses on the load. In the present configuration these values are approximately 100 ns. Methods of reducing rise/fall time on the LEBT electrodes are discussed. Results of simulation and comparative measurements of the original and upgraded system on the test stand are presented. Furthermore, the effect of these changes on reliability degradation caused by arcing in the LEBT structure is discussed.
\end{abstract}

\section{I.INTRODUCTION}

The Spallation Neutron Source (SNS) linac H- beam is injected into the storage ring, accumulated there and then extracted from the ring to the target. The Low Energy Beam Transport (LEBT) chopper system provides deflection of part of the beam during a 1-ms macro-pulse by applying high voltage (HV) pulses of $+/-2.5 \mathrm{kV}$ to deflecting electrodes, creating gaps between the beam mini-pulses. This is required in order to minimize beam loss during ring extraction [1]. The width of the HV pulse can be varied but it is limited due to the reasons shown in [2]. Partial deflection losses depend on transients in the system. Improvements of the rise/fall time of the HV pulse at the electrode help to provide more effective chopping and reduce beam losses in the linac and the ring extraction area. Also, reduction of the chopper rise/fall time permits longer beam pulses since the minimum chopper pulse width (and hence the maximum time the beam can pass through un-chopped) is determined by these values.

This paper describes modifications to the LEBT chopper network which achieves the required HV pulse parameters on the deflector. Measurement results of the original and upgraded configuration taken on a test stand are presented. Upgrades to the system which were implemented two years ago and described in [3] minimize possible risks of damage after the changes to the new configuration. The test results of the arcing of the electrode event are shown also.

\section{LEBT CHOPPER SYSTEM CONFIGURATION}

The SNS LEBT chopper design was described in [4]. The chopper incorporates four $+/-2.5 \mathrm{kV}$ pulsed power supplies (pulsers) which drive four quadrants of an electrostatic focusing lens floating at $-50 \mathrm{kV}$. Figure 1 presents a simplified block-diagram of the system.

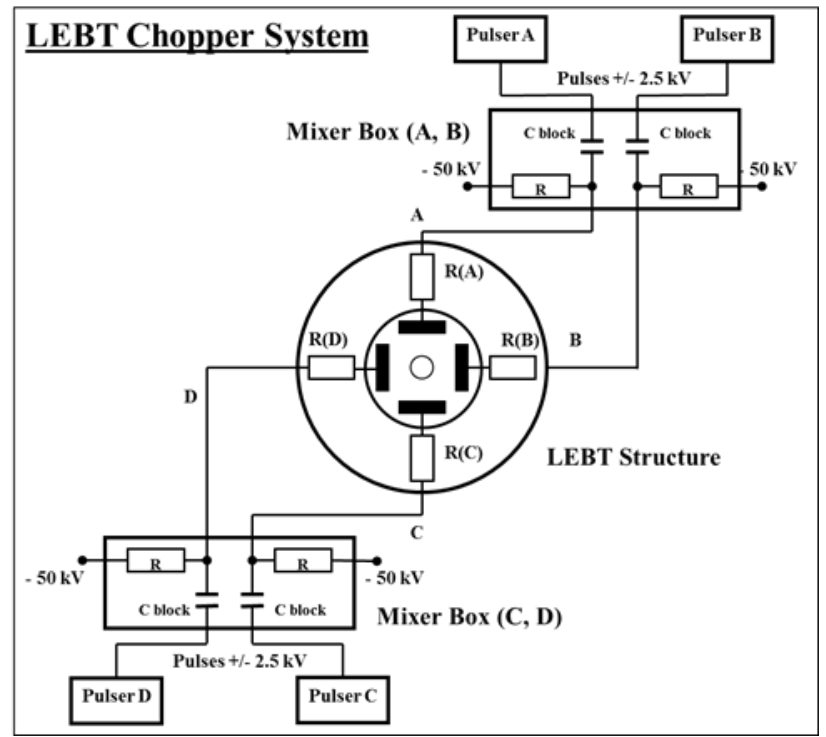

Figure 1. LEBT chopper system block-diagram.

\footnotetext{
*The work at Oak Ridge National Laboratory, which is managed by UT-Battelle, LLC, was performed under contract DE-AC05-00OR22725 for the US Department of Energy
} 
A more detailed block-diagram of one channel in Fig. 2 shows three major units: pulser, mixer box and LEBT structure, which are interconnected via short-lengths of coaxial cable.

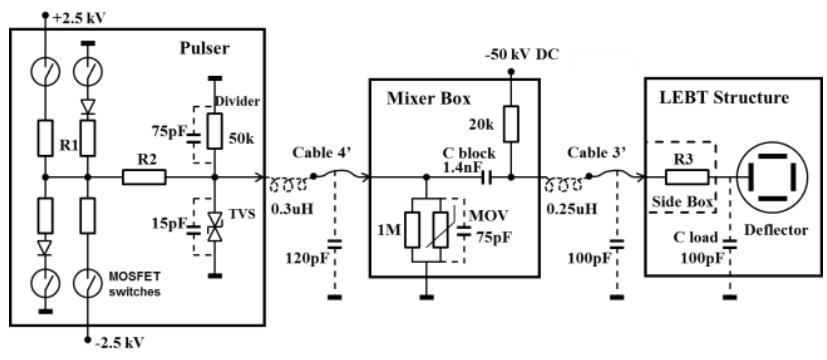

Figure 2. "Pulser-Mixer Box-LEBT Structure" network block-diagram.

The key components of the pulser are the solid-state pull-up and pull-down MOSFET switches connected in such a way as to create a series of positive and negative $\mathrm{HV}$ pulses at the output. The coupling capacitor $\mathrm{C}_{\text {block }}$ inside the oil-filled mixer box, whose value is selected to pass the HV pulses, allows delivery of ground referenced $+/-2.5 \mathrm{kV}$ pulses to the deflecting electrode. The deflector is biased at a potential not greater than $-50 \mathrm{kV} \mathrm{DC}$ from external power supply. There are three different currentlimiting series resistors in this network: R1 is located in each switch leg, R2 is installed at the pulser output and $\mathrm{R} 3$ is inside the air-filled side box of the LEBT structure. These resistors also create a voltage divider during the load fault event (arcing in the deflector) when the energy stored in the blocking capacitor discharges through them. The network contains over-voltage protection elements such as transient voltage suppressors (TVS) and varistors (MOV), a voltage divider for voltage monitoring, HV feed-through connectors and wires.

The deflecting electrode presents a capacitive load of approximately $80 \mathrm{pF}$. Rise/fall times of the rectangular $\mathrm{HV}$ pulse at the deflector depend on the performance of the pulser itself, values of the series resistors R1, R2 and R3 and the network configuration. All of the elements of the circuit have their own parasitic and stray capacitances and inductances and impact deflector's response time. Some of the measured and calculated parasitic values are shown in Fig. 2.

\section{RISE AND FALL TIME IMPROVEMENT}

\section{A. History}

The pulser provides rise and fall times of less than $35 \mathrm{~ns}$ with no load connected at its output. It is obvious that the values of the series resistors R1, R2 and R3 and stray capacitances basically determine the rise/fall times on the capacitive load. Originally, the values of the resistors R1 and $\mathrm{R} 2$ installed in the pulser were $13.4 \Omega$ and $75 \Omega$, respectively. Initially, the resistor R3 was located inside the mixer box and had a value of $400 \Omega$ or even $750 \Omega$ at different periods of time. This was varied in an attempt to eliminate MOSFET switches failures, thought to be caused by over-current during an arc event.

Later, the value of $\mathrm{R} 1$ was reduced to $11.3 \Omega$. The value of the resistor R3 was reduced to $330 \Omega$, and it was relocated into the side box of the LEBT closer to the deflector to eliminate cable capacitance and thus reduce the $\mathrm{RC}$-constant in the last section of the circuit.

In the past the failure rates of the LEBT chopper pulser have been very high. An extensive study of pulser failure root causes was done in 2010. As a result, multiple changes have been designed to mitigate these failures. The results of study and upgrades have been described in [3]. Since the upgrades were implemented in 2011, there have been no pulser failures, allowing further work on rise/fall time improvement.

\section{B. Measurement Results}

To simulate a real SNS LEBT system and provide accurate measurements, a test stand has been assembled which includes a pulser, modified mixer box, and $\mathrm{HV}$ capacitor load.

Measurements of the pulse on the load revealed the rise/fall time values are close to $100 \mathrm{~ns}$ in the original configuration where R1, R2 and R3 were $11.3 \Omega, 75 \Omega$ and $330 \Omega$, respectively. The goal was to achieve rise/fall times less than $50 \mathrm{~ns}$ by reducing the values of these resistors. All three resistors are to be reduced proportionally to ensure the voltage divider ratio and thus the voltage distribution in the circuit is not changed during a load fault.

New values of the resistors R1, R2 and R3 have been chosen. They are $7.5 \Omega, 25 \Omega$ and $200 \Omega$ respectively. Figure 3 presents the pulse waveform for the original and upgraded configurations. One can observe efficient reduction of the rise/fall times.

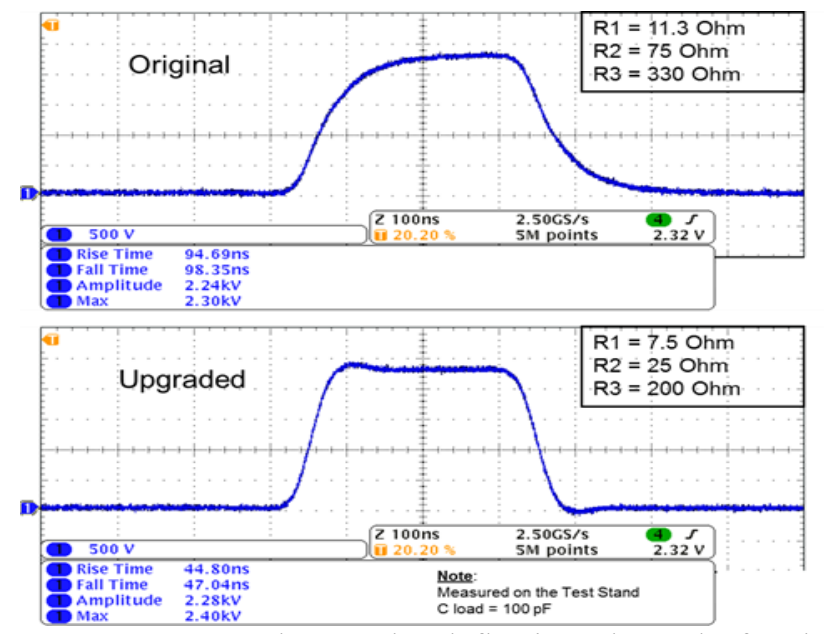

Figure 3. HV pulse on the deflecting electrode for the original and upgraded configurations. 
The rise and fall times have been reduced from approximately $100 \mathrm{~ns}$ with the original resistors to less than 50 ns with new resistors installed; a 50\% improvement.

\section{Pulse Width Limitation}

Limitations of the pulse width in the system were presented and described in detail in [2]. It was shown that the minimum pulse width that ensures full deflection of the beam in the original configuration is 200 ns. A reduction of the pulse rise/fall times can help shorten the pulse in the LEBT chopper and still achieve flat top at full amplitude.

Measurements were performed on the test stand to demonstrate this. The input trigger pulse width was decreased in $15 \mathrm{~ns}$ steps and the pulse on the load was monitored. Figure 4 shows the result: the amplitude of the pulse flattop begins to decrease when $T$ trigger becomes shorter than $200 \mathrm{~ns}$ in the original configuration, and it does not change until trigger becomes less than $170 \mathrm{~ns}$ in the new configuration. This means that the minimum achievable pulse width on the LEBT chopper deflector is reduced by $30 \mathrm{~ns}$, and the beam pulse width in the linac can be increased by approximately $4 \%$, resulting in higher proton beam energy delivery to the neutron target.

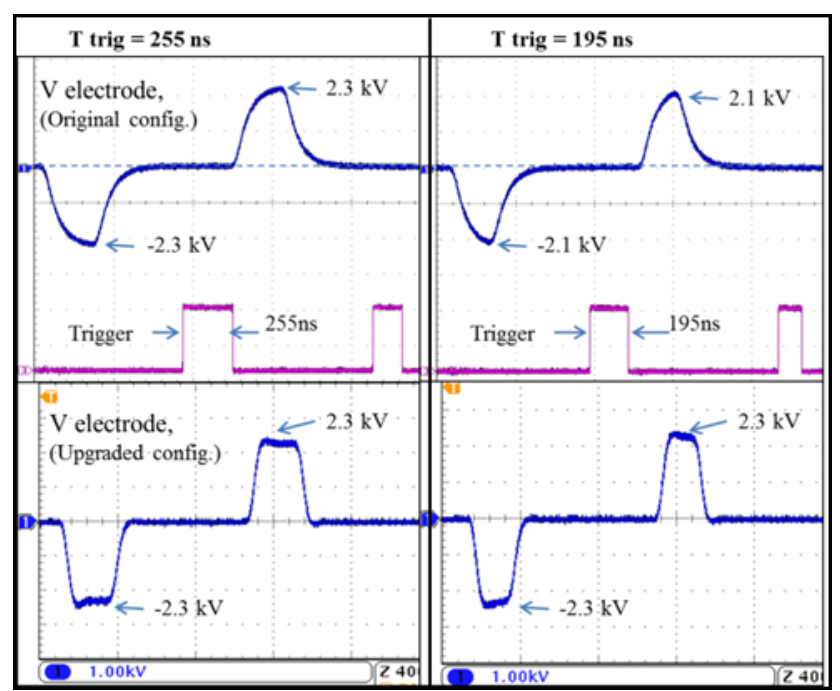

Figure 4. Waveforms of the HV pulse on the load while changing trigger pulse width.

\section{PROTECTION OF MOSFET SWITCH}

Each pulser's HV switch consists of eight MOSFETs connected in series. Each MOSFET has maximum breakdown voltage rating of $1000 \mathrm{~V}$, pulsed current of 56 A, dissipation power of $400 \mathrm{~W}$ and maximum junction temperature of $150^{\circ} \mathrm{C}$. Three types of faults may occur during operation: over-voltage, over-current and overheating. Each of these events may happen when the load is shorted, and could result in MOSFET failure if there is no protection.

As discussed in [3], failures of the MOSFET switches and blocking diodes in the pulser were correlated to arcing in the LEBT structure. Four major changes were made in 2011: reduced arc rate, improved voltage sharing, improved voltage clamping and modified gate latching [3]. These upgrades resulted in zero switch failures during last two years operation. Figure 5 shows the arc and failure rate since 2007 .

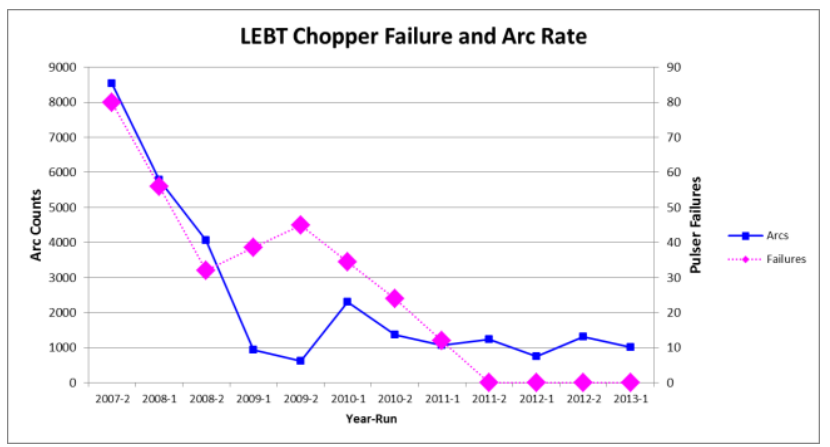

Figure 5. Pulser failure rate and numbers of arc events.

\section{A. Normal Operation}

During normal operation at $+/-2.5 \mathrm{kV}$ the MOSFET current is a half-sine wave during the rising and falling edges of each pulse. The amplitude of the pulse depends on the total series resistance in the circuit. The total series resistance of the "pulser - mixer box - load" circuit is reduced by approximately $80 \%$ in the new configuration. The peak current through the MOSFETs will increase accordantly, but does not exceed the maximum current rating. Since the duration of the current pulses are of tens of nanoseconds only and the duty factor is very low, there is not an over-heating issue.

\section{B. Shoot-Through Condition}

A shoot-through condition, when all MOSFETs in the positive and negative $\mathrm{HV}$ legs, or in the $\mathrm{HV}$ and grounding legs of one polarity, are closed simultaneously, can happen due to errant MOSFET gate drive. As has been mentioned above, modification in the gate drive circuit essentially excluded this possibility. In addition, the electronics in the pulser contains an over-current protection circuit with a response time less than $200 \mathrm{~ns}$. This mitigates the MOSFET over-current and overheating failures. The R1 value limits the peak shoot-thru current to $2 \times 2.5 \mathrm{kV} \div 2 \times(\mathrm{R} 1+\mathrm{Ron})$, where Ron is the OnState resistance of eight MOSFETs, or $178 \mathrm{~A}$. This is about three times the maximum pulsed current rating of the MOSFETs but only persists for up to $200 \mathrm{~ns}$ and is therefore safe for reliable operation of the pulser.

\section{Arcing in the Structure}

The blocking capacitor is charged to the $-50 \mathrm{kV}$ DC bias voltage. In the case of arcing in the LEBT structure 
the load capacitance is shorted and the blocking capacitor discharges through the series resistors and MOSFETs.

Voltage distribution across three series resistors is proportional to the resistor's values. Table 1 shows the voltage divider ratio for the original and upgraded configurations. The total resistance value is the sum of three values $(\mathrm{R} 1+\mathrm{R} 2+\mathrm{R} 3)$.

Table 1. Voltage divider ratio

\begin{tabular}{|c|c|c|c|c|c|}
\hline \multicolumn{2}{|c|}{ Resistor } & R1 & R2 & R3 & $\begin{array}{c}\text { Total } \\
\Sigma\end{array}$ \\
\hline \multirow{2}{*}{ Old } & $\begin{array}{c}\text { Value, } \\
\boldsymbol{\Omega}\end{array}$ & 11.3 & 75 & 330 & 416.3 \\
\cline { 2 - 6 } & $\begin{array}{c}\text { Ratio, } \\
\text { \% }\end{array}$ & 2.7 & 17.9 & 79.4 & 100 \\
\hline \multirow{2}{*}{ New } & $\begin{array}{c}\text { Value, } \\
\mathbf{\Omega}\end{array}$ & 7.5 & 25 & 200 & 232.5 \\
\cline { 2 - 6 } & $\begin{array}{c}\text { Ratio, } \\
\text { \% }\end{array}$ & 3.2 & 10.8 & 86 & 100 \\
\hline
\end{tabular}

As shown in Table 1, the voltage divider ratio between three resistors has been changed slightly. This will result in increased voltage drop across R3 during the arcing, but it will not exceed resistor's rating $60 \mathrm{kV}$. The voltage drop across two resistors R1 and R2 together, which adds to the voltage drop across the switch and appears at the pulser output, will be reduced in the new configuration.

During the arcing the drain current increases rapidly and can exceed the maximum pulsed current rating. If the operating point goes above the Ohmic region "knee" on the MOSFET's output characteristic, any further increase in drain current results in rise in "ON" resistance, limiting the current [5]. The voltage drop across the MOSFET rises, increasing the conduction loss, but the power dissipation is very small because the arcing in the LEBT structure is limited to a single event. The RC timeconstant in the discharging circuit is less than $350 \mathrm{~ns}$, so the junction temperature never exceeds the $150^{\circ} \mathrm{C}$ limit.

\section{D. “Arc Test" on the Test Stand}

The arcing is a sporadic event. It can occur during positive pulsing, negative pulsing, between pulses or between bursts. Since the pulse duty factor is less than $1.5 \%$ the probability of an arc during pulsing is low. To ensure the MOSFET switches can survive arcing in the structure, a test was performed on the test stand to simulate arcing.

A simplified diagram of the circuit for the case when the arcing event occurs between pulses is presented in Fig. 6. The $-50 \mathrm{kV}$ power supply was connected to the load via a $20 \mathrm{k} \Omega$ current-limiting resistor which is installed inside the mixer box. Over-voltage protection elements (TVS and MOV) were installed in the circuit to simulate the existing configuration. Two capacitors, $\mathrm{C}_{\text {block }}$ and $\mathrm{C}_{\text {load }}$ are charged to $-50 \mathrm{kV}$. Then the load capacitor $\mathrm{C}_{\text {load }}$ was momentary shorted, with the blocking capacitor discharging through three series resistors and the closed
MOSFET switch. Two voltage probes were installed to monitor voltages at the switches and at the pulser's output (test points TP1 and TP2 in Fig. 6).

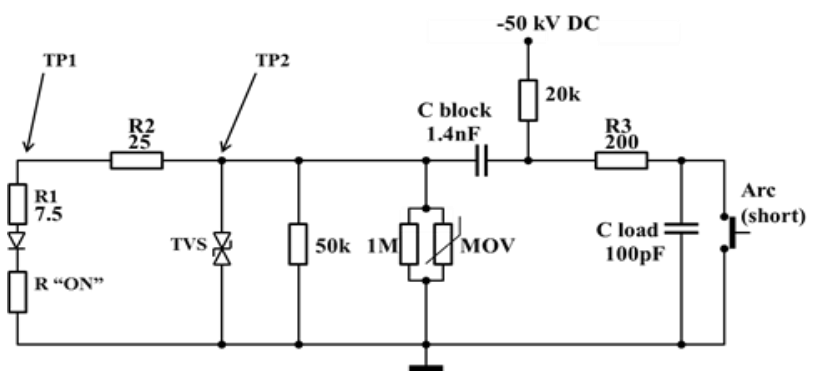

Figure 6. Simplified block-diagram for "Arc Test" measurements.

Figure 7 shows an example of the typical voltage waveforms. The total breakdown voltage of the fast transient voltage suppressors (TVS) at the output of the pulser is approximately $3.5 \mathrm{kV}$. One can observe TVS clamping effect on the upper picture. The over-shoot voltage at this point (TP2) is higher than the clamping voltage because the inductance of the wires produces the over-shoot voltage. This spike is very short (less than 20 $\mathrm{ns)}$ and its amplitude does not exceed $5 \mathrm{kV}$. The amplitude of the first voltage spike at the MOSFET switches (TP1) is much lower than their voltage rating of $8 \mathrm{kV}$.

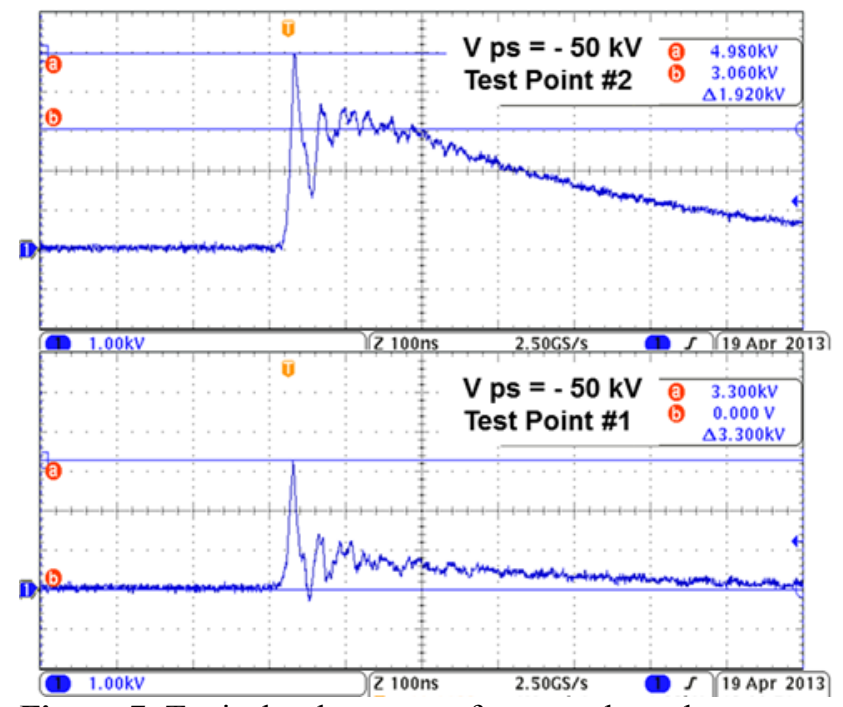

Figure 7. Typical voltage waveforms at the pulser output (upper) and at MOSFET switches during "arc test" on the test stand.

The worst-case scenario is if the arc occurs during a positive pulse at the output because the additional +2.5 $\mathrm{kV}$ applies to the opened switch in the grounding leg, but even in this case the peak voltage will be less than the maximum voltage rating of the MOSFET array. 


\section{SUMMARY}

Proportional reduction of the values of three series resistors in the LEBT chopper circuitry will provide:

- $50 \%$ improvement of rise/fall times of the HV pulse on the deflector (T rise/fall $\leq 50 \mathrm{~ns}$ );

- $4 \%$ extension of beam mini-pulse width achievable in the system;

- Adequate MOSFET switches protection.

Fault voltage levels will not exceed those observed with the original configuration, so system reliability is not expected to decline as a result of making the resistor changes described.

\section{REFERENCES}

[1] S. Nath, J. Billen, J. Stovall, H. Takeda, L. Young, LANL, Los Alamos, NM 87545, USA, D. Jeon, S. H. Kim, SNS/ORNL, Oak Ridge, USA, and K. Crandall, Tech Source, Santa Fe, NM 87501, USA, "Beam Behavior through the SNS Chopper System", in Proc. of the LINAC 2002 Particle Accelerator Conference, Gyeongju, Korea, 2002, pp. 130-132.

[2] V. V. Peplov, R. B. Saethre, "SNS LEBT Chopper Pulse Width Limitation", in Proc. of the 2012 IEEE International Power Modulator and High Voltage Conference, San Diego, 2012, pp. 700-703.

[3] R. B. Saethre, V. V. Peplov, "SNS LEBT Chopper Improvements", in Proc. of the 2012 IEEE International Power Modulator and High Voltage Conference, San Diego, 2012, pp.704-707.

[4] J. W. Staples, J. J. Ayers, D. W. Cheng, J. B. Greer, M. D. Hoff, A. Ratti, "The SNS four-phase LEBT chopper", in Proc. of the 1999 Particle Accelerator Conference, New York, 1999, pp. 1961-1963.

[5] Jonathan Dodge, P.E., "Power MOSFET Tutorial", Advanced Power Technology Application Note, APT0403 Rev. B, 2006. 